\title{
Erratum zu: Die klinische Anwendung von humanen induzierten pluripotenten Stammzellen
}

Sara Gerke, Jochen Taupitz, Claudia Wiesemann, Christian Kopetzki, und Heiko Zimmermann

Erratum zu:

S. Gerke et al. (Hrsg.), Die klinische Anwendung von humanen induzierten pluripotenten Stammzellen, Veröffentlichungen des Instituts für Deutsches, Europäisches und Internationales Medizinrecht, Gesundheitsrecht und Bioethik der Universitäten Heidelberg und Mannheim 48, https://doi.org/10.1007/978-3-662-59052-2

Folgende Korrekturen wurden ausgeführt:

1. Das Kapitel „Herausforderungen innovativer Gewebemedizin aus unternehmerischer Sicht" von Michael Harder wurde ursprünglich als nicht Open Access-Kapitel veröffentlicht, wird hiermit aber in eine Open Access Publikation unter der CC BY 4.0-Lizenz geändert; die Angabe des Copyright Holders wurde dementsprechend in „@Der/die Autor(en)“ angepasst.

2. Für das Kapitel „Naturwissenschaftliche, ethische und rechtliche Empfehlungen zur klinischen Translation der Forschung mit humanen induzierten pluripotenten Stammzellen und davon abgeleiteten Produkten“ wurde die Angabe des Copyright Holders von ,®CThe Author(s)“ in ,@Der/die Autor(en)“ korrigiert.

Die aktualisierte Version des Kapitels finden Sie unter:

https://doi.org/10.1007/978-3-662-59052-2_5

https://doi.org/10.1007/978-3-662-59052-2_11 